\title{
AQUATICS reconstruction software: the design of a diagnostic tool based on computer vision algorithms
}

\author{
Andrea Giachetti ${ }^{1}$ and Gianluigi Zanetti ${ }^{1}$ \\ CRS4 - Parco Scientifico e Tecnologico POLARIS, Edificio 1, Loc. Pixina Manna, 09010 Pula (CA), \\ Italy $\{$ giach, zag $\}$ @crs 4 . it
}

\begin{abstract}
Computer vision methods can be applied to a variety of medical and surgical applications, and many techniques and algorithms are available that can be used to recover 3D shapes and information from images range and volume data. Complex practical applications, however, are rarely approachable with a single technique, and require detailed analysis on how they can be subdivided in subtasks that are computationally treatable and that, at the same time, allow for the appropriate level of user-interaction. In this paper we show an example of a complex application where, following criteria of efficiency, reliability and user friendliness, several computer vision techniques have been selected and customized to build a system able to support diagnosis and endovascular treatment of Abdominal Aortic Aneurysms. The system reconstructs the geometrical representation of four different structures related to the aorta (vessel lumen, thrombus, calcifications and skeleton) from CT angiography data. In this way it supports the three dimensional measurements required for a careful geometrical evaluation of the vessel, that is fundamental to decide if the treatment is necessary and to perform, in this case, its planning. The system has been realized within the European trial AQUATICS (IST-1999-20226 EUTIST-M WP 12), and it has been widely tested on clinical data.
\end{abstract}

\section{Introduction}

Computer vision (like computer graphics) provides many techniques and algorithms that can be used to recover $3 \mathrm{D}$ shapes and information from images range and volume data: pixel (voxel) classification techniques, isosurface extraction methods, etc. Some of them have been validated, even if only by using particular data under particular conditions, others are already used in industrial applications, while many others have only been proposed without the support of a large amount of experimental results. To choose the algorithm class that is best suited to a specific problem is indeed an important step in developing medical (as well as industrial) applications. Furthermore, a typical medical application may involve different sub-tasks, each with its own peculiarity, and often there is not a single reconstruction technique powerful enough to deal with all of them. The application designer needs, therefore, to find specific solutions for each subtask and to combine these solutions in a, possibly, user friendly and effective software tool.

In the following sections we will show how we combined four different state-of-the art computer vision tools in a system that helps vascular surgeons and interventional radiologists in the preoperative evaluation of the abdominal aorta. The system is capable of completely recover, with a fast and mostly automatic method, the geometrical structure of abdominal aorta from CT scans of the abdominal region, and to present clinicians with an interactive, measurable, 3D model of the vessel and ancillary structures.

The reconstruction of the aorta is very important for the evaluation of Abdominal Aortic Aneurysms (AAA): the precise measurement and evaluation of their geometrical parameters is fundamental to estimate rupture risk and to plan surgical or endovascular interventions, see $[2,5$, $20,24,25]$.

Following the requirements coming from experts in vascular surgery, we developed specialized methods adapted to the different structures to be recovered, that is vessel lumen, vessel skeleton, plaques, thrombus. The result of our work are new flexible computer vision algorithms, and a user friendly software tool that includes all these algorithms and it can be used to obtain, in a fast and interactive way, a full vessel reconstruction. 


\section{Vessels structures and CT}

As an introduction and motivation to the following sections, we give here a brief description of the application context and image data features. As mentioned above, the goal of our work was to provide a tool for endovascular intervention planning capable of accurate pre-operative measurements based on a model of the vascular tree, of the arterial lumen, thrombus structures and calcified plaques obtained from the segmentation of CT images.

Arteries, e.g., the aorta, are vessels conducting the bulk of oxygenated blood flow to the body. The lumen of a vessel is the region where the blood flows; its volume is not perfectly constant in big elastic vessels due to pressure variation during the cardiac cycle, but it can be approximately considered constant for our application. Aneurysms are local dilation of the vessels greater than $150 \%$ of normal diameter. They are probably caused by atherosclerosis or cystic medial degeneration. If their diameter is larger than $55 \mathrm{~mm}$. the probability of their rupture - an event causing death in most cases - is extremely high and aneurysms of that size must be absolutely treated. In the aortic aneurysms the lumen is often separated from the vascular tissues by thrombus. Thrombus is an aggregation of blood factors, primarily platelets and fibrin with entrapment of cellular elements, frequently causing vascular obstruction at the point of its formation. Other structures often surrounding the aorta, and that it is necessary to localize in order to avoid problems during surgery or endovascular treatment, are calcified plaques i.e., fat, cholesterol and mineral deposits that can develop on the inside of arterial walls. Their presence may cause problems in fixing prostheses [20] or inserting catheters inside the vessel.

Segmentation is performed on acquired CT scans. CT angiography is the standard for pre operative aortic diagnosis. It creates a 3D set of X-ray absorption maps coded as gray levels with a resolution higher than any other 3D acquisition technique. The greatest limitations to CT angiography are partial volume effects, which result in gradual attenuation transitions between adjacent structures: models reconstructed must always be considered affected by an error at least equal to maximum between the slice thickness or the slice spacing of the CT acquisition. A typical slice thickness for these diagnostic protocols is between 1 and $2 \mathrm{~mm}$.

It is not possible to discriminate vessel tissues from $\mathrm{CT}$ data, but it is possible to have a clear delineation of the lumen by injecting a contrast medium in the blood. By doing so, the gray level in the image, corresponding to a well defined Hounsfield value (the X-ray attenuation), becomes sufficiently distinguishable from the surrounding. Thrombus and calcified plaques have Hounsfield values very close to the level of other structures, but they can be usually identified, even though small calcifications cannot be located because they are typically masked by volume effects.

\section{Surface extraction techniques}

Our task involves the estimation of three different surfaces enclosing three different structures: vessel lumen, vessel walls with thrombus regions, calcified plaques. There are three main classes of algorithms in literature to recover surfaces from voxelized volume.

Contour based surface extraction: the volume of the CT data is cut with series of planes, possibly perpendicular to the vessel direction ${ }^{1}$, and, on each plane, contours of the lumen intersection are evaluated using edge following, region growing or snake algorithms. Finally, contours are connected in tubes and tubes are connected in trees. This method gives an immediate estimation of the skeleton as connection of contour mass centers.

Iso-surface extraction with Marching Cubes: marching cubes [16] give a surface that separate voxels with gray value higher and lower than a defined threshold by defining look up table associating surface element to voxel neighborhoods. The extraction is done usually on classified or pre-processed data. The surfaces extracted are not connected, require post processing for mesh simplification and smoothing, even if a lot of work has been done to improve the quality of the extracted surfaces since the algorithm has been introduced;

\footnotetext{
${ }^{1}$ This is not an easy task and so it is usually assumed, as a first approximation, that the vessel is oriented along one of the $\mathrm{CT}$ scan axis
} 


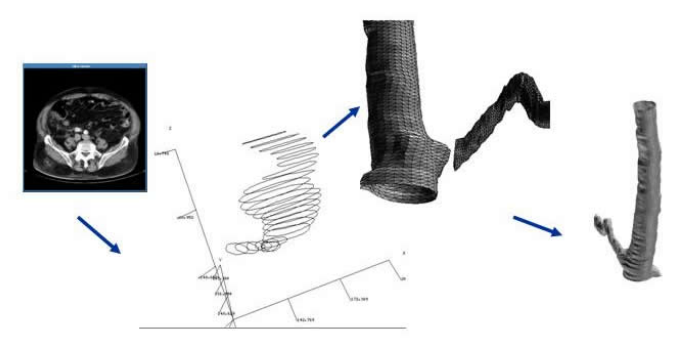

Fig. 1. Steps in contour based lumen reconstruction: cutting planes are first selected, contour computed with a snake algorithm, triangulated tubes are generated and finally joined after finding their intersection.

Deformable models: surfaces in 3D can be defined with implicit or parametric functions and adapted locally to the images. Surveys of this class of methods can be found in $[18,19]$. Three dimensional snakes (inflatable balloons), and front propagation method [22] are examples of, respectively, a topology preserving / not preserving method in this class.

We now proceed in the analysis of advantages and drawbacks of each of these methods when applied to our reconstruction tasks.

\section{Lumen reconstruction}

\subsection{Contour based surface extraction}

For the lumen reconstruction all the techniques could be applied, we therefore compared their results. We found, however, that is difficult to recover complete vessel lumen geometries using a $2 \mathrm{D}$ contour based approach, because vessels have foldings and irregularities along their length. We implemented for comparison sake a 2D contour based method with the contours defined on 2D images obtained by tri-linear interpolation on arbitrary, user selected, planes across the dataset. Starting from contours series we then build tubes and assemble them in a vessel tree structure using geometrical engines like GTS [12]. This approach was dropped because it requires strong user interaction to obtain reliable results on all data sets, especially in the reconstruction of arterial tree bifurcations and it is not generally applicable.

\subsection{Iso-surface extraction with Marching Cubes}

Given the difficulties encountered with 2D contour based methods, we moved to a fully 3D method, based on first binarizing the volume data between a connected vessel lumen region and the rest, and then extracting the vessel surface as an iso-surface. To binarize the data we used a region growing method based on front propagation, that was initialized with a point inside the region of interest and whose growth was continued until it encountered voxels with gray level differing from the reference value more than a threshold. Holes were then filled using a topological closing algorithm. After data binarization, we applied a standard marching cubes algorithm. The resulting surfaces needed then to be regularized and simplified with triangle decimation. This approach, however, did not give reliable results: surfaces are good only in the case of well contrasted lumen with negligible noise and artifacts.

\subsection{Deformable models}

In literature, model based approach $[15,1]$ based on an initial ridge extraction at different scales (hard in noisy and complex situation) or surface based approaches $[17,11]$ are the methods proposed mainly for this task.

Among all the possible deformable model algorithms we selected an explicit method deforming a closed surface. The reasons for this choice are the following. 

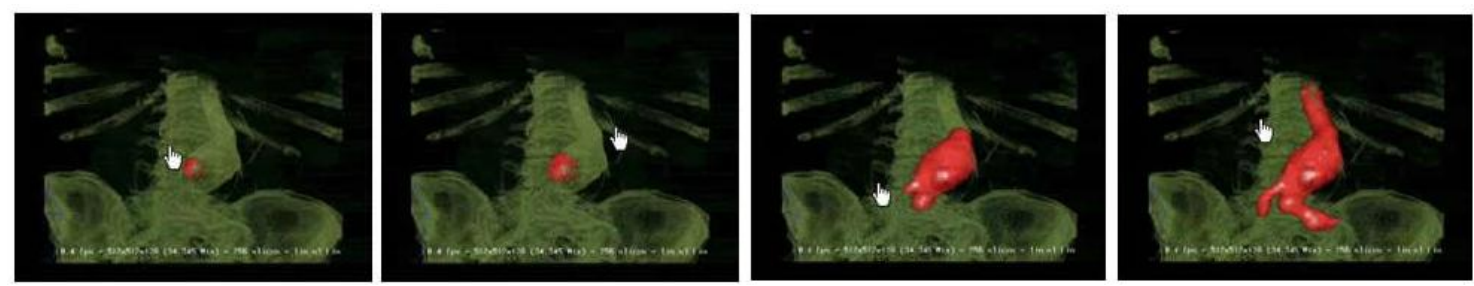

Fig. 2. Four successive steps of a balloon growth visualized over the corresponding volume-rendered CT data set.

- Fast computation: the use of implicit methods, finite elements, etc. makes evolution slower.

- Topology preservation: it is assumed that the lumen is an unique surface not separable into isolated parts.

- Sensitivity to noise: elastic forces keep the contour smooth and are less influenced by local noise or artifacts.

We have implemented a deformable surface algorithm, called "fast simplex mesh balloon", specifically designed for this task. It is based on the Simplex Mesh geometry introduced in [9]. A generic Simplex Mesh is a N dimensional mesh with N+1 connectivity. The Simplex Mesh we use is therefore a closed surface mesh composed by nodes each connected with three neighbors. Nodes move under the influence of an inflating force directed along the surface normal vector, an elastic smoothing force described ("surface orientation continuity constraint") and two image forces. The first, a deflating force directed against the surface normal, compensates the inflating force when the local average of the gray level differs from the internal value more than a fixed threshold. The second, an edge attraction force, pushes nodes toward the maximum of the gray level gradient modulus in the neighborhood. To avoid a large variance in simplex sizes, the latter are controlled by a simplex merging/splitting algorithm that is invoked every so many iteration steps. Our simplex algorithm is adaptive and optimized for the extraction of tubular structures: the maximum face size is not a global value, but is proportional to the local curvature. To speed up the computation, nodes that have already reached the desired border (i.e., an edge with the gray level which differs more than a fixed thresholds from the internal value) are labeled as fixed. The algorithm generates surfaces well adapted to complex structures, and the computation is fast because at each step only the free "front" of the surface in the tubular structure is moved. The simplex mesh is composed by polygons that are not necessarily planar and cannot be easily rendered, and therefore the final simplex mesh is converted to its "Dual" form (i.e., a new triangulated mesh with nodes in the center of the faces and connections corresponding to the simplex edges) to have a smooth triangulation [9]. The surface is usually initialized as a small sphere inside the lumen and then undergo the evolution determined by the forces applied. The user can control the maximum number of iterations to be performed, force parameters and the maximum and minimum size of the polygons. Autointersections are prevented by an appropriate test.

Surface evolution can be automatically stopped when the nodes do not move relevantly, but in our application it is usually stopped manually in order to avoid the detection of structures that are not interesting for our application.

The final procedure is described by the following lines of pseudo-code.

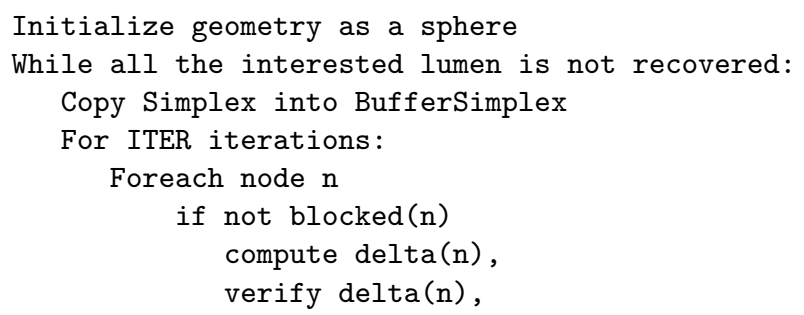




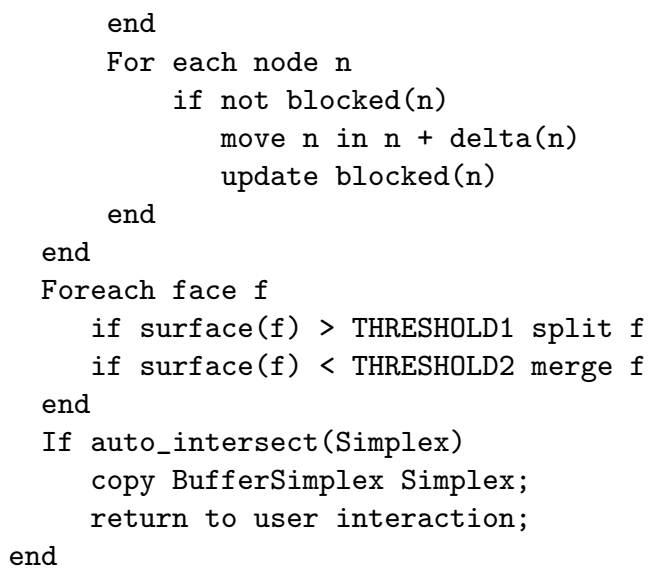

This approach gave the more precise results requiring the minumum user interaction and was therefore chosen for the application.

\section{Calcifications reconstruction}

The recovery of the calcium boundaries is an ideal application of isosurface extraction. For this task, in fact, it is simply required to extract boundaries of voxels with a well defined HU value. No topological properties or support for measurements are needed. We applied therefore the well known "marching cubes" algorithm [16] with a threshold chosen to represent the calcification boundaries. Even in this case, however, it is necessary to customize the procedure and to consider some peculiarities of the problem. This threshold cannot be set at the $120 \mathrm{HU}$ suggested, for example, in [13], because this value is lower than the level of the contrast medium injected in the lumen. We put the threshold usually at about $320 \mathrm{HU}$, considered by many the lowest value in plaques [10].

We also limited the isosurface computation to a region defined by the user by selecting a bounding box.

Finally, if geometries must be exported or used for Internet applications or fast rendering there is the possibility of reducing the number of triangles with standard decimation routines provided by the GTS library [12].

\section{Thrombus reconstruction}

It is difficult to automatically identify thrombus. Deformable surface methods are not useful since thrombus is not contrasted and its density, 20-50 HU, is too close to those of fat and of the noncontrasted vessels, and there are too many edges inside it due to calcifications. The best option seems, therefore, to use a 2D approach based on computing series of snakes or inflated balloons $([14,8]) .2 \mathrm{D}$ contours can be easily controlled, constrained and manually corrected by the user (see Fig.4.1). 3D tubular surfaces can, as seen before, recovered by joining contours with an algorithm finding point to point correspondences and, since ion this case the thrombotic regions are usually reasonably "straight", the approach provides reasonably good results. De Bruijne et al., [6], have recently proposed what amounts to the first specialize thrombotic vessels segmentation method. In their work they report a semi-automated procedure that uses contour based methods with model based constraints based on Active Shape Models. In our opinion, the use of model based constraint methods is limited by the great variability of physiological shapes and the limited training set generally used to build shape models. De Bruijne et al. considered also this fact and relaxed the constraints by adding synthetic covariance in the deformation model they built. 

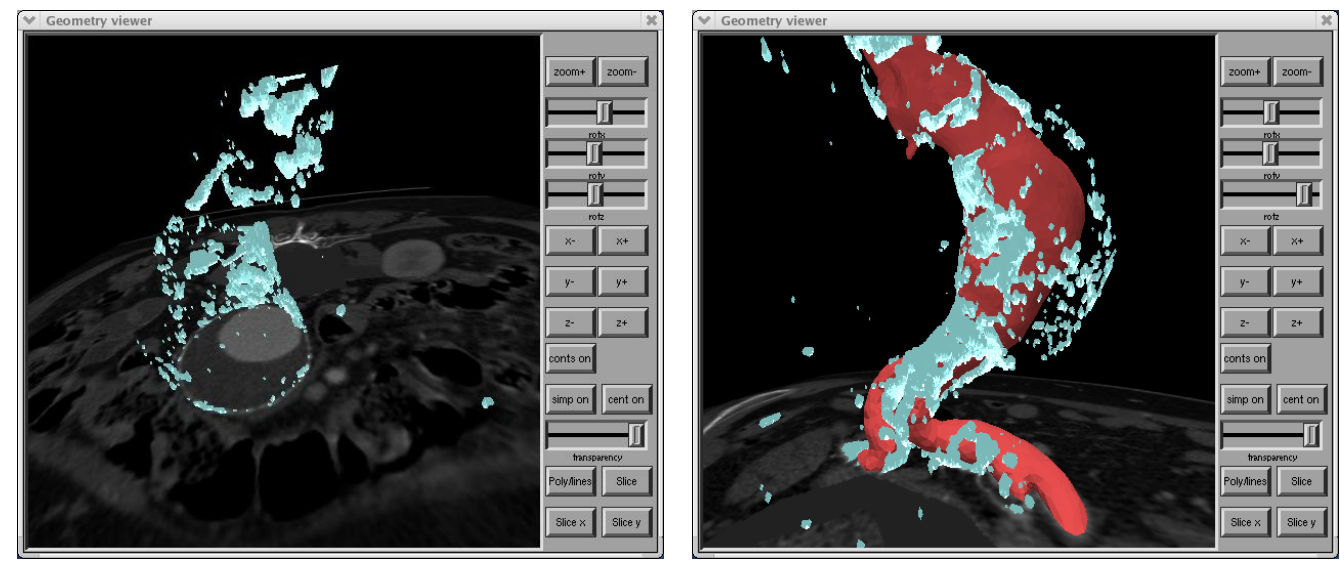

Fig. 3. Left: external surfaces of calcified regions extracted with marching cubes at 320 HU. Right: Lumen and calcium surfaces superimposed to the CT scan

We completely dropped the statistical approach, using still a constrained contour based method. We introduced contour evolution constraints by using a simple Fourier snake, like those described in [23], with a weighted correction aiming at moving the contour close to the correct boundaries.

The algorithm works directly on CT slices. We know in advance the lumen geometry and its skeleton and thus we do not need to recover the vessel structure with other model based approaches as in [6]. The reference model used is therefore described by:

$$
\begin{gathered}
f_{x}(i, t)=a_{0}+\sum_{k=1}^{n} a_{k} \sin (k 2 \pi i / N) \\
f_{y}(i, t)=b_{0}+\sum_{k=1}^{n} b_{k} \sin (k 2 \pi i / N)
\end{gathered}
$$

where $i, t, N, n$ are, respectively, the point label, the time step, the number of points defining the snake and is highest order in the Fourier expansion.

At each iteration, the snake points are driven by standard internal forces, a rejecting force depending on a threshold on the difference between local $\mathrm{HU}$ value and thrombus $\mathrm{HU}$, and a specially designed edge force. The latter is a force directed along the derivatives of the image gradient that is active only if the edge shape is compatible with the border of a thrombotic region. This activation is done by computing a weight $w$ for each border point as follows:

- Define a search space locally perpendicular to the curve (we use \pm 4 pixels)

- Count in the search space internal pixels in the range of thrombus values

- Count in the search space external pixels in the range outside thrombus values

if both counts are larger than 1 , the edge is considered a possible thrombus limit and the value of $w$ is set to 1 (0 otherwise). The final formula is

$$
F_{e}(i)=-\alpha \cdot w \cdot \nabla|E(\boldsymbol{p}(i))| \operatorname{step}(|\nabla E(\boldsymbol{p}(i))|-\text { threshold })
$$

At each iteration step, we compute the displaced points $\boldsymbol{s}(i)$ by applying as finite differences the standard inflated balloon forces [8]. At each iteration of the point update, we then compute the truncated Fourier approximation of $\boldsymbol{s}(i, t), \boldsymbol{f}(i, t)$, i.e., the constrained snake, and then obtain the final point position as a linear combination of the free and constrained displacement, using, for the free displacements, weights proportional to the previously computed "scores":

$$
\boldsymbol{p}(i, t+1)=\gamma \cdot w \boldsymbol{s}(i, t+1)+(1-\gamma \cdot w) \boldsymbol{f}(i, t+1)
$$



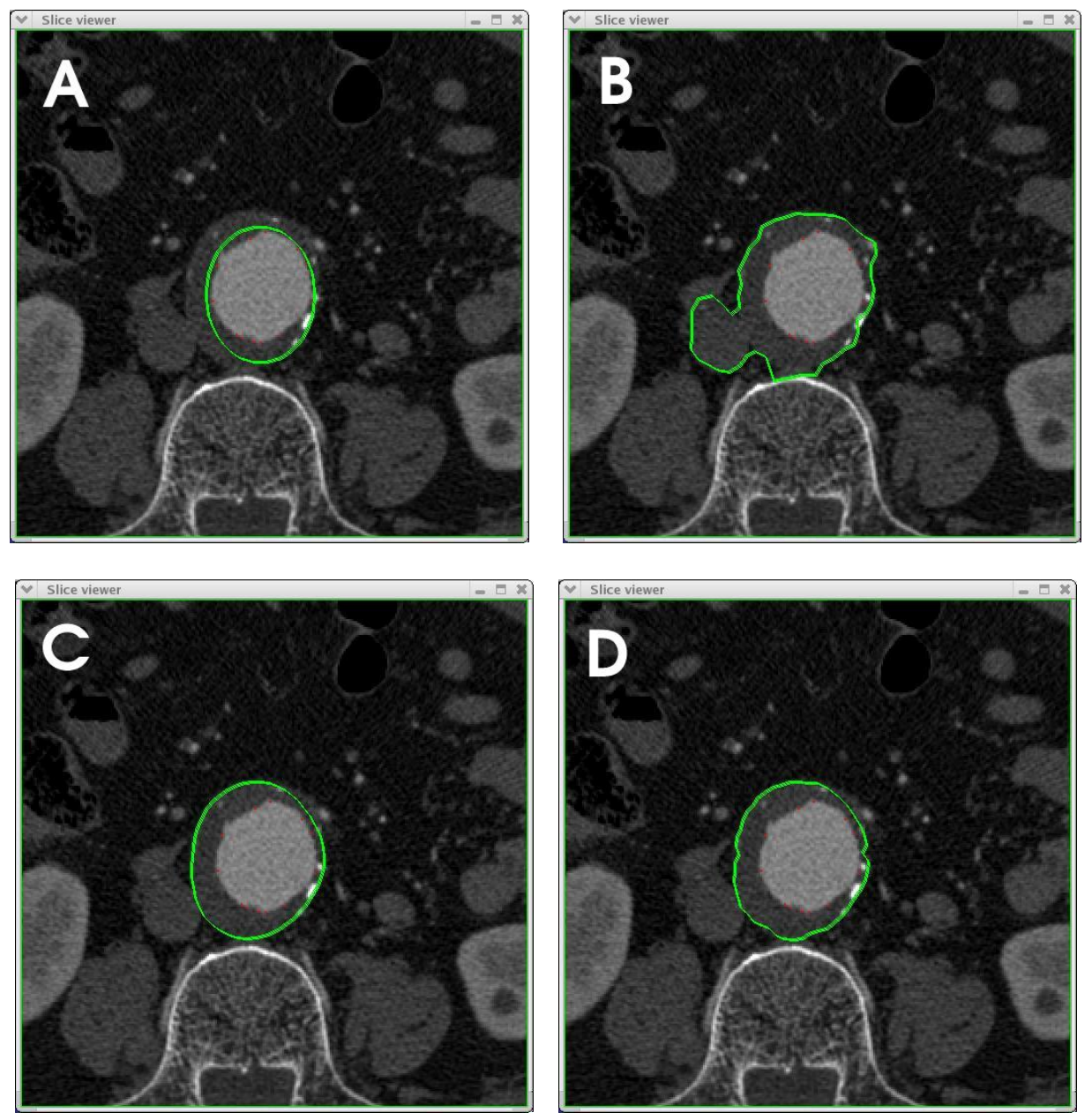

Fig. 4. Examples of thrombus segmentation. A: automatic initialization outside the lumen surface. B: unconstrained evolution: problems are created in regions lacking information. C: Fourier constrained evolution. D: filtered evolution: the border is well adapted to borders near the edges.

where $\gamma$ is an estimate of the accuracy in the thrombus boundary delineation. The "filtering" step close to edges limiting the thrombotic region. We found this procedure sufficient in order to avoid some false detections due to the poor contrast between the thrombus HU and the environment.

In some cases image information is too poor and it is not possible to eliminate completely the possibility of a wrong contour placement. User interaction is thus required for correction. We, therefore, concentrated our efforts on finding a procedure with fast extraction capability but whose behavior could be easily controlled by user-interaction. The procedure works as follows:

- First contour is started automatically as an ellipse outside the lumen.

- Standard filtered evolution is launched.

- Result is visually inspected. If something is wrong the user can immediately correct the contour, both by changing segmentation parameters, and manually moving points.

- A simple menu allows the transition to the next image. Here the contour can be initialized by duplicating the previous one. If it is not considered appropriate the user can either choose an ellipsoid outside the lumen or a contour can be easily drawn manually.

- Continuing the extraction of the following contours, the user can also see the 3D surface generated in an OpenGL window together with the other structures and textured planes representing coronal axial or sagittal sections. 
When all the contours are computed, they are resampled with the same number of nodes and connected in a tubular geometry. If it is considered necessary, it is possible to extract several tubular parts and join them in bifurcated structures. The procedure described allows a sufficiently fast thrombus recovery, since the need of performing manual corrections of the contour is reduced at less than $5 \%$ of the $\mathrm{CT}$ images analyzed and it is extremely simple.

It is clear that the thrombus extraction remains the most difficult part of the complete reconstruction due to its poor contrast. Fig. 4 shows a typical segmentation procedure on a single slice: Fig.4A shows the initialization as an ellipse outside the lumen, Fig.4B the result of a nonconstrained evolution, Fig.4C the result of a completely constrained evolution, while Fig.4D the result of a "filtered" one. Fig. 5 shows a screen shot of the thrombus segmentation procedure. Fig 6 shows a complete thrombus segmentation superimposed to a CT section.

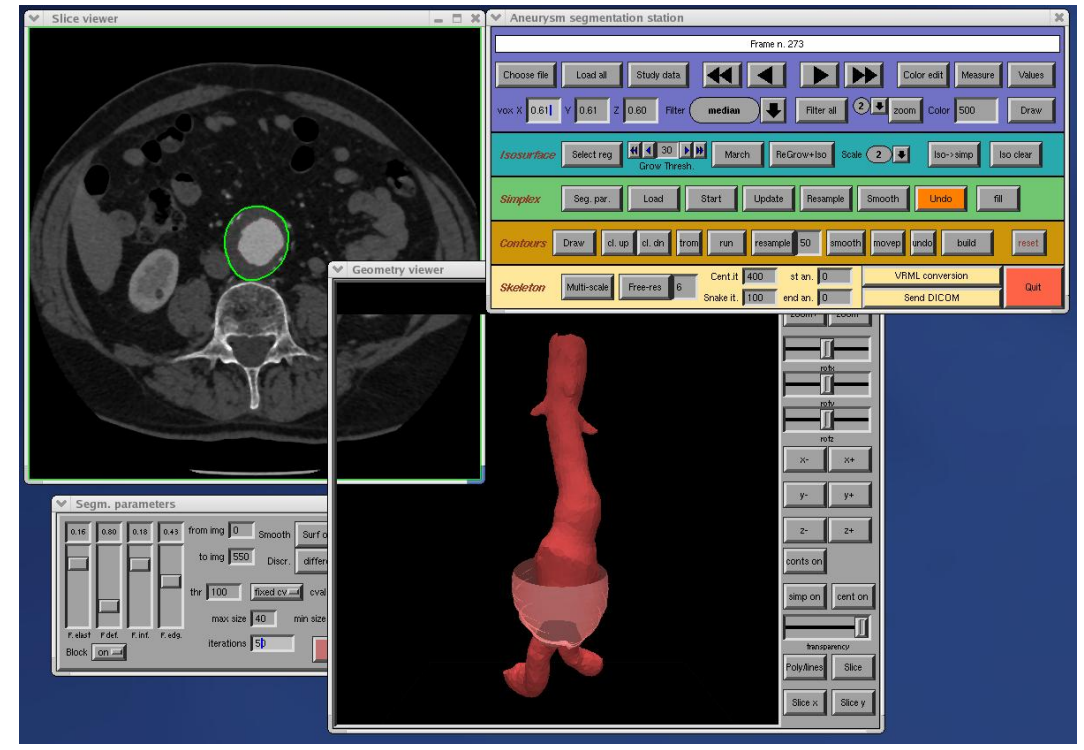

Fig. 5. Screenshot taken during the thrombus segmentation.

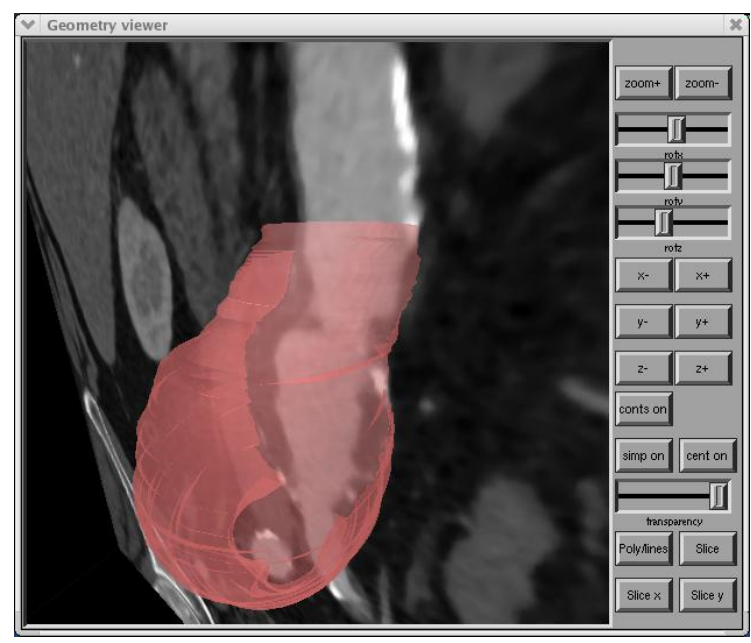

Fig. 6. An extracted thrombus superimposed to a sagittal section of the abdomen. 


\section{$7 \quad$ Skeletonization of the lumen}

Skeleton extraction is fundamental to capture the local direction of the "vessel tube" and the networked structure of the whole organ. In 3D things are much more complicated than in $2 \mathrm{D}$, where skeletons can be easily extracted with a medial axis transform [4]. The "medial axis" in 3D is a surface. In 3D it is even difficult to give a definition of skeleton. For voxelized geometries it is usually defined as a set of one voxel thick lines with particular properties, i.e. they are centered, they are connected, they are smooth. Two main techniques have been proposed to extract this kind of lines from classified voxels (methods based on surfaces have been also proposed [26] but with less reliable results): topological thinning [21] or voxel coding based $[27,7,3]$. Considering advantages and drawbacks, we finally chosen and implemented an improved voxel coding method that uses a multi-scale approach and a snake-based regularization. Voxel coding algorithms have been recently introduced and seem the simplest and more general methods able to give fast and sufficiently accurate results. Considering that a distance map from the border (BSC,boundary seeded code) in the 3D case is not sufficient to extract one voxel thick skeletons as in the 2D case, the idea of these methods is to compute first paths inside the volume, and then center them using the distance map. To extract the paths another voxel coding is defined, called "Single Seeded Code" (SSC) or "Distance from Seed", measuring the distance of volume points from a seed voxel. Taking as starting points local maxima of the SSC with high values, paths are extracted searching for voxels with lower SSC in the neighborhood. When the voxel has been found, it is added to the centerline and then a new voxel with lower SSC is searched around it. The procedure is stopped when the seed is reached or when the line is close to a previously extracted one. The extracted lines are approximately "shortest" paths joining the starting point and the seed. Paths are depending on the metric used to compute the SSC and on the search strategy. The skeleton defined in this way have some nice properties: it is composed by lines, i.e. by lists of connected point, it capture the network structure of the vessel and its points are inside the volume. Two desired properties can be, however, still missing: centering and smoothness. The usual approach presented by authors to center the lines is derived by Zhou and Toga and consists of the following steps:

- For each point of the skeleton, find the cluster of voxels with the same SSC, connected with that point.

- Find the voxel of the cluster with maximum BSC

- Move the centerline point to the position of that voxel

Results are not always satisfactory. This procedure can give reasonable skeletons in the case, for example, of vascular structures with approximately constant radius, but are strongly dependent on the position of the seed and on the shape of the object to be skeletonized.

We have improved this algorithm by introducing a multi-scale snake based regularization, and it is now faster and less influenced by local structure. Our multi-scale snake based regularization is driven by the distance from border map. The latter is acting as an energy field and the resulting elastic forces makes the lines centered and smooth. With our method we obtain results compliant with our requirements: i.e., continuous curves connected in a tree structure and locally centered in the volume. The algorithm works as follows:

- Find the internal region on the full dataset by region growing inside the previously extracted surface.

- Create a low resolution binarized dataset labeling as internal all the voxel at the low resolution including an internal voxel at the high resolution. The user interaction is therefore only in the choice of the desired initial resolution and in the selection of the starting point.

- Compute the centerline at the low resolution, i.e. compute the BSC and the SSC at the low resolution, and find the tree structure.

- Compute the boundary seeded code map at the high resolution, called BSCH

- Move each skeleton point to the high resolution voxel location corresponding to the maximum of the $\mathrm{BSCH}$ inside the low resolution voxel.

- Resample and regularize the line with the snake algorithm. 


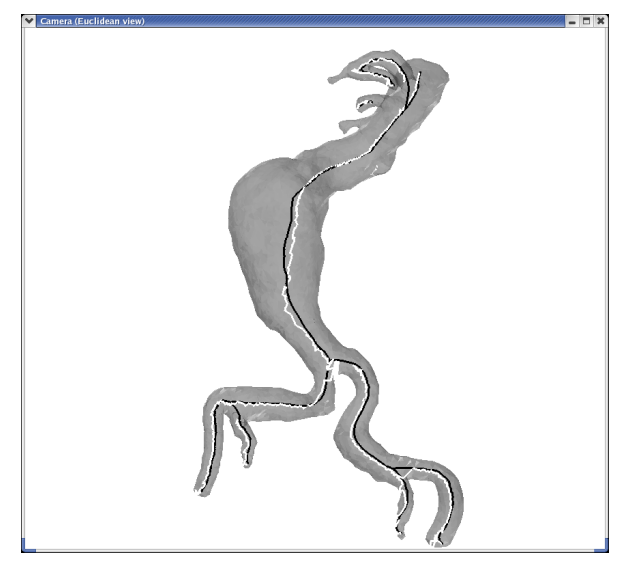

Fig. 7. Example of skeleton extraction. White line: Shortest path at high resolution. Black line: Final snake-based centerline extracted.

- Go on finding the other branches, joining their last point to the closest point of the previous lines. Lines shorter than a fixed threshold are removed.

We found that in our application this revised method is extremely fast and reliable. Fig.7 shows an example of the procedure.

\section{Validation}

The reconstruction software described in the previous sections was used in the AQUATICS trial within the European Project IST EUTIST-M. The basic idea of AQUATICS was to build a prototype of a service for surgical centers able to provide in few hours after a CT scan and, using the web as delivery mechanism (see Fig. 9, 3D measurable models of the aorta in order to support the collaborative planning of endovascular treatment. The system evolved during the project and it is now complete, enabling the reconstruction of a complete model in about one hour. During the project two different technicians performed reconstructions using the tool and three clinical specialists used the models to measure a set of parameters necessary for endovascular procedure planning. More than 40 patient specific aortic models have been reconstructed and models of a synthetic phantom have been recovered from CT scans for validation. AQUATICS measurements are compatible with phantom's true data and patient data measurements done manually by radiologists using standard methods. The t-test showed a very good correlation between the measurements obtained on phantom with the Aquatics system and the true measurements of the phantom $(p<0.0001)$ demonstrating the reliability of the system. The correlation between observers was also tested with the Spearman rank test and again a statistical significant correlation was proved $(p<0.0001)$. Similar results were obtained on measurement performed on models reconstructed by different operators. Therefore the system proved to be reliable and its results reproducible.

\section{Discussion}

In order to achieve a complex diagnostic task, i.e. supporting a fully 3D geometrical quantitative evaluation of Abdominal Aortic Aneurysm, we selected and customized four computer vision techniques able to recover different parts of the model and used them as ingredients to build an integrated application with an user friendly interface, that can be used by trained radiologists in ordinary conditions. Experimental results show that the two main goals of the applications have been reached with this approach: in fact complete aortic reconstruction with all the four interesting 

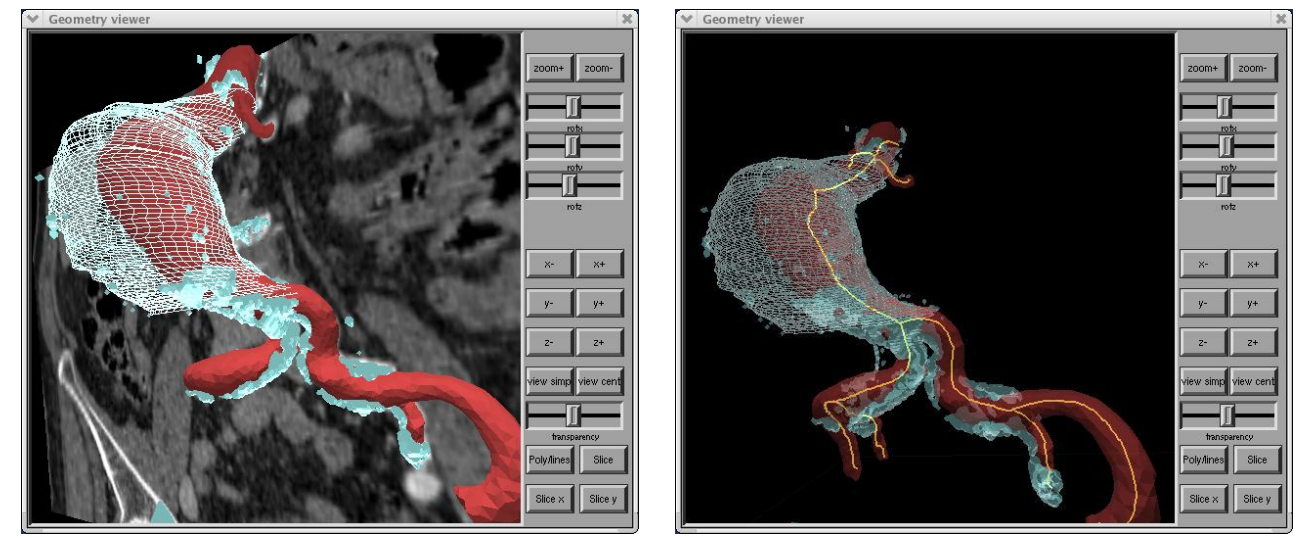

Fig. 8. Left: Reconstruction of the three different (lumen, thrombus, calcium) obtained with the different algorithms superimposed to the CT data. Right: Same model with the automatically extracted skeleton.

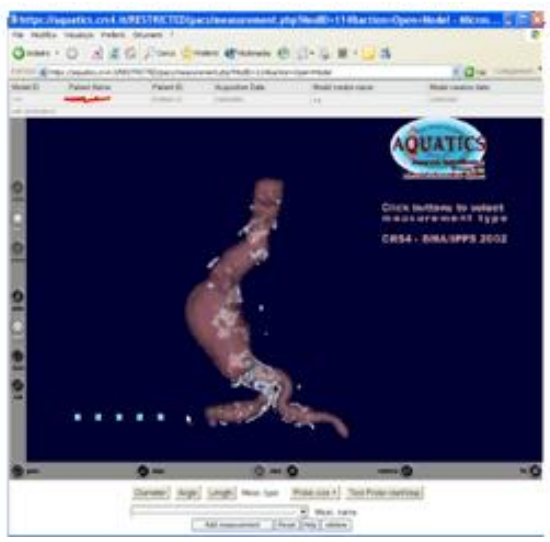

Fig. 9. Example of a completely reconstructed aneurysm viewed on a web interface allowing measurements.

structure can be reconstructed by a trained operator within a "reasonable time" (approx. 1 hour) the clinical evaluations performed on the model was found sufficiently precise compared with other methodologies during the validation trial.

The experience acquired during the system development suggested us that to the design of the complex application that can be effectively used in a clinical environment requires

- A good knowledge of the state of the art and a careful analysis of the users requirements, since many factors have an influence on how algorithms are chosen. Efficiency and precision are not the only factors to be considered, simplicity and easiness of use must be also taken into great account.

- The development of simple, but powerful user interfaces that allow the user to have immediate visual feedback for the result of all the algorithms applied. Not all the computer algorithms can give reliable results in all conditions and this must be always known by the user that must have the possibility to check the results.

We believe that image processing and computer vision tools will be applied to many new tasks and become widely used in hospitals and clinical institutions if well designed applications will be developed and a correct information about the power and the limits of these techniques will be brought to the medical community.

Acknowledgments: We would like to thank Emanuele Neri, Irene Bargellini, (University of 
Pisa), Ammar Mallhoui (University of Innsbruck) and Massimiliano Tuveri for data selection and medical analysis. Enrico Bertolazzi and Gianmarco Manzini for providing their simplex library. Work was supported by the EUTIST-M EU funding (IST-1999-20226).

\section{References}

1. S.R. Aylward,"Initialization, Noise, Singularities and Scale in Height Ridge Traversal for Tubular Object Centerline Extraction" IEEE Trans. on Medical Imaging, 21,2 61-75 (2002)

2. K. M. Baskin et al., "Volumetric Analysis of Abdominal Aortic Aneurysm", Medical Imaging 1996: Physiology and Function from Multidimensional Images, Eric A. Hoffman, Editor, Proc. SPIE 2709, p. 323-337 (1996).

3. I. Bitter, A. Kaufman and M. Sato(2001) " Penalized-Distance Volumetric Skeleton Algorithm ," IEEE Transactions on Visualization and Computer Graphics, Vol. 7, No. 3, July-Sept. 2001, pp. 195-206

4. H. Blum, "A transformation for extracting new descriptors of shape," Proc. Symp. Models for the Perception of Speech and Visual Form, MIT Press, Cambridge, MA, pp. 362-380, 1967

5. Blankesteijn, J.D., "Imaging techniques for endovascular repair of abdominal aortic aneurysm". Medica Mundi 44/2 November 2000

6. M. de Bruijne et al, "Active shape model based segmentation of abdominal aortic aneurysms in CTA images". Medical Imaging 2002, Image Processing Proc. SPIE Vol 4684 pp. 463-474 (2002)

7. D. Chen et al, " A Novel Approach to Extract Colon Lumen from CT Images for Virtual Colonoscopy ," IEEE Transactions on Medical Imaging, Vol. 19, No. 12, December 2000, pp. 1220-1226.

8. L.D. Cohen and I. Cohen, "A finite element method applied to new active contour models and 3D reconstructions from cross-sections" Proc. of 3rd Int. Conf. on Comp. Vision, pp. 587-591 (1990).

9. H. Delingette, "Simplex meshes: a general representation for 3d shape reconstruction", in CVPR94, pp. 856-859, 1994.

10. P. Felkel, "Segmentation of Vessels in Peripheral CTA Datasets", VRVis Center Technical report TR-VRVis-2000-008, Vienna, Austria, 2000

11. A.F. Frangi et, al., "Quantitative Analysis of Vascular Morphology from 3D MR angiograms: in Vitro and In Vivo Results" Magn Res. in Med. 45,311-322 (2001)

12. S.Popinet, Project: The GNU Triangulated Surface Library http://sourceforge.net/projects/gts

13. I Isgrum et al., "Automatic detection of calcifications in the Aorta from abdominal CT scans", Excerpta Medica ICS1256, proc. CARS 2003, pp. 1037-1042 (2003)

14. A. Kass, A. Witkin and D. Terzopoulos, "Snakes: Active contour models," Int. J. of Comp. Vision 1, 321-331 (1988).

15. K. Krissian et al. "Model Based Multiscale Detection and Reconstruction of 3D vessels" INRIA Sophie Antipolis Report n.3442 (1998)

16. W.E. Lorensen and H. E. Cline, "Marching cubes: a high resolution 3D surface construction algorithm". In M.C. Stone ed., SIGGRAPH '87 Conference Proceedings, pp. 163-170 (1987).

17. D. Magee, A. Bulpitt, E. Berry, "3D Automated Segmentation and Structural Analysis of Vascular Trees Using Deformable Models" Proc. IEEE Workshop on Variational and Level Set Methods in Computer Vision, 2001.

18. T. Mc Inrey and D. Terzopulos, "Deformable models in medical image analysis, a survey" Medical Image Analysis, 1(2): 840-850, 1996

19. Montagnat, J, Delingette, H, and Ayache, N, "A review of deformable surfaces: topology, geometry and deformation," Image and Vision Computing, vol. 19, pp. 1023-1040, 2001.

20. J.C. Parodi, J.C. Palmaz, H.D. Barone, "Transfemoral intraluminal graft implantation for abdominal aortic aneurysms" Ann Vasc Surg 1991;5:491-499.

21. R.J.T. Sadleir and P.F. Whelan, "Colon Centerline Calculation for CT Colonography using Optimised 3D topological thinning" Proc. IEEE 3DPVT, pp.800-803 (2002)

22. J.A. Sethian, "Level Set Methods \& Fast Marching Methods: Evolving Interfaces in Computational Geometry, Fluid Mechanics, Computer Vision \& Materials Science, Cambridge University Press, 1996

23. L.H. Staib and J.S. Duncan, "Boundary Finding with Parametrically Deformable Models," IEEE Transactions on Pattern Analysis and Machine Intelligence, 14(11):1061-1075, 1992.

24. J.D. Santilli and S. M. Santilli, "Diagnosis and treatment of Abdominal Aortic Aneurysms" American Family Physician 56:4 (1997)

25. Tillich M, Hill BB, Paik DS, Petz K, Napel S, Zarins CK, Rubin GD., "Prediction of aortoiliac stent-graft length: comparison of measurement methods." Radiology 2001 Aug;220(2):475-83

26. Masayuki Hisada, Alexander G. Belyaev, Tosiyasu L. Kunii, 3D Voronoi-Based Skeleton and Associated Ninth Pacific Conference on Computer Graphics and Applications (PG'01) Surface Features

27. Y. Zhou and A. W. Toga, "Efficient skeletonization of volumetric objects", TVCG, vol. 5, 1999. 